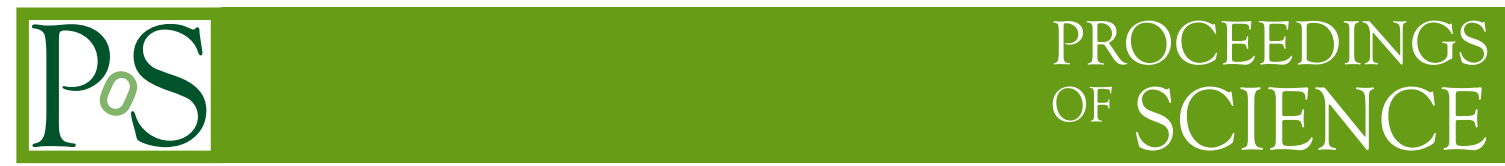

\title{
Observing the Dawn of the Universe
}

\section{Jose Miguel Rodríguez Espinosa*}

Instituto de Astrofísica de Canarias, La Laguna, Spain

E-mail: jmr.espinosa@iac.es

The dawn of the Universe is elusive. Observations of the early Universe are hampered by neutral gas at the beginning of the universe as well as by the faintness of the recently formed sources. We explain the way these sources are typically observed, and propose to observe the dawn of the Universe with a micro-satellite equipped with a near IR camera. These will provide the large field necessary, and enough contrast for observing line emission.

Frontier Research in Astrophysics - II

23-28 May 2016

Mondello (Palermo), Italy

${ }^{*}$ Speaker. 


\section{Summary}

It is commonly assumed that the Universe started producing Population III stars and galaxies around $\mathrm{z} \sim 30$. These were high luminosity pure hydrogen stars. Later on these stars could assemble into galaxies which would accreting more and more material and/or merge, to produce larger galaxies. Galaxies that eventually were able to re-ionised the Universe. In this paper I will explain how astronomers typically observe these early galaxies. I will also discuss our plan to observed these early sources with a micro-satellite equipped with a near IR camera.

\section{Introduction}

Observations of high redshift galaxies provide a better understanding of the large scale structure formation of the Universe. Thus, observing the galaxies as far back as the time of their assembly is ideal for studying the early evolution of the Universe. However, detecting these high redshift galaxies is challenging due to their low surface brightness. Nevertheless, novel observation techniques combined with large telescopes and their instruments allow us to detect many high-z galaxies, especially Lyman $\alpha$ (Ly $\alpha$ ) Emitters (LAEs) and Lyman Break Galaxies (LBGs) (e.g. Bouwens et al. (2010, 2015), Ellis et al. (2013), Giavalisco et al. (2004), McLure et al. (2010), Ouchi et al. (2003); Ouchi et al. (2008, 2010), Shapley et al. (2003), Steidel et al. (1999), and Taniguchi et al. (2005)).

LAEs and LBGs have long since been theorised to be observable up to the Reionisation Epoch (Meier 1976; Partridge \& Peebles 1967). Studying these galaxies is crucial to understanding how the complete reionisation of the intergalactic medium (IGM) in the early Universe was realised. The luminosity functions of high-z LAEs and LBGs are established to be steeper than the low-z populations. This leads to the conclusion that the majority of the ionising photons responsible for reionisation of the intergalactic neutral hydrogen is produced from the young stellar populations of low mass star forming galaxies (e.g. Erb (2015), Dressler et al. (2011, 2015), Henry et al. (2012), and Yan et al. (2010)).

\section{Observational strategies}

LAEs and LBGs are primeval, star forming galaxies with strong Ly $\alpha$ emission the former, the latter possessing significantly stronger UV-continuum than the former (Haiman \& Spaans 1999). Because of this, early universe sources have typically been observed either using a narrow-band filter or through the drop-out technique. The use of a narrow band filter increases the contract of the Lyman $\alpha$ line, allowing the detection of Lyman $\alpha$ emitters (LAEs). The drop-out technique is rather used for the Lyman Break Galaxies (LBGs). This technique is based in the Lyman continuum break, beyond $912 \AA$. This drop in an already faint source produced a lack of detection in a given broad-band filter, while the source is seen in redder filters (Figure 2)

LAES and LBG are distinguishable because the former do not have any Lyman continuum, though they do have prominent Ly $\alpha$ line emission. Thus their behaviour is very different in the presence of neutral hydrogen gas or dust. Indeed the Ly $\alpha$ line is immediately absorbed by dust, and resonantly scattered bu neutral Hydrogen. 


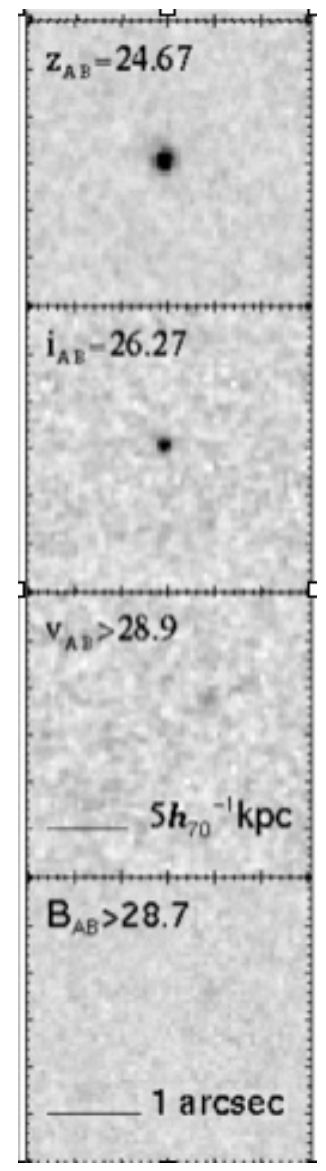

Figure 1: Detection of a LBG. using the drop-out technique. In this case it would be a V drop-out, as the signal is lost in the V broad-band filter.

\section{The re-ionisation of the Universe}

It is well known that the present day universe is fully re-ionised. This was not so after recombination, at a redshift of $\sim 1000$. The Universe was re-ionised at $z \sim 5.5$ ([7]). How the Universe was re-ionised is still a matter of debate. Observations point towards a steepening of the Luminosity function of star forming sources at high redshifts. Figure ??, shows luminosity functions at redshifts 7, 8 and 9. It can be seen that these Luminosity functions do get steeper as redshift increases. However, it is also seen in this figure that the number of sources with which this figure has been done is very small. With that caveat in mind, what that figure implies is that the number of low luminosity sources increases steeply with redshift. So it may be the case that the re-ionisation of the Universe was achieved by this large population of low luminosity sources.

\section{Observing the dawn of the Universe}

Observing the dawn of the universe may not be easy as the universe was mostly neutral then. Theoretical studies carried out in our group ([19]) reveal that the Universe may have been previously reionised, to a large extent, by PopIII stars by redshift 10 or so. These stars would have 


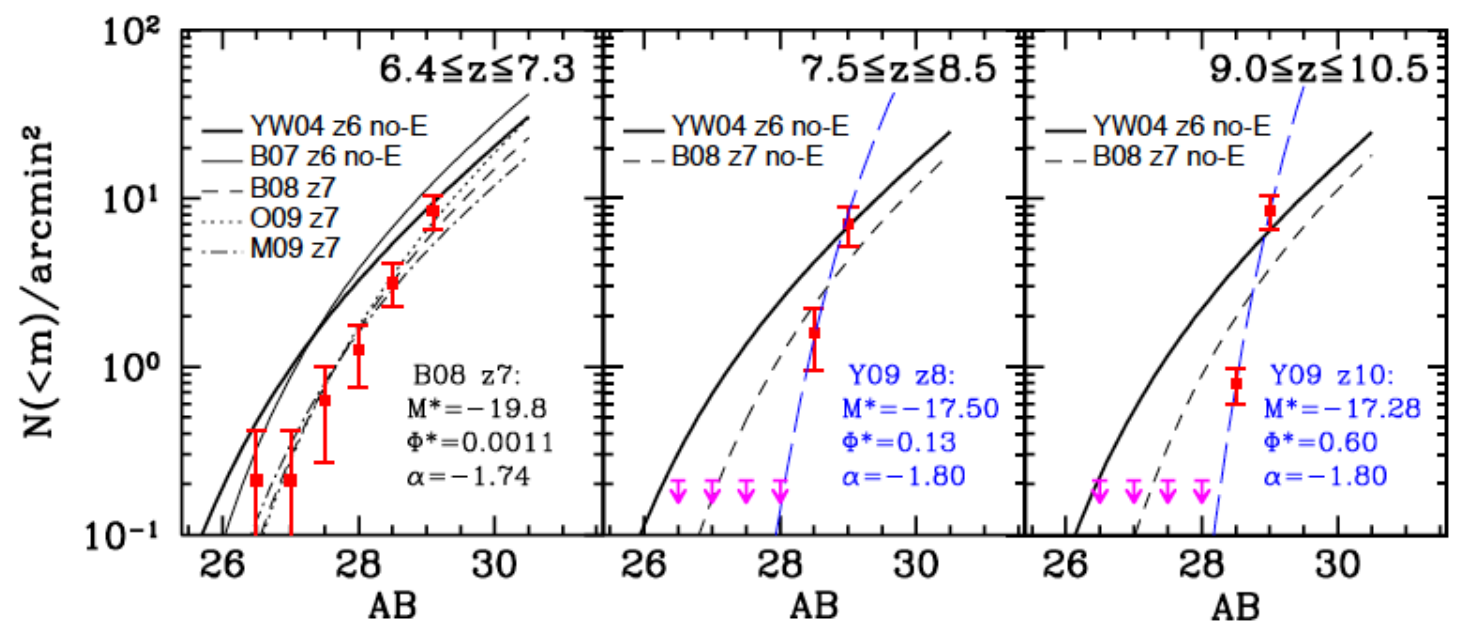

Figure 2: Luminosity functions at $z=7, z=8$ and $z=9$ from ([21]). Note how hey get more steep as the redshift increases.

petered out by then. Hence the universe may have started to become neutral again. There is however a window in which the ionisation status of the Universe was almost complete, offering a window for the possible observations of the first galaxies. Those that were responsible for completing the full re-ionisation of the Universe.

Therefore, our plan is to use a micro-satellite to search blindly for these first sources. A microsatellite with a small telescope and a near infrared camera has the advantage of offering a very large field of view (ideal for blind searches). Besides, the IR camera allows using a narrow band filter plus a broad band one, to search for the Ly $\alpha$ emission line at redshift of about 10, where the Universe was mostly ionised by Pop III stars. The satellite will be dedicated so large integrations will be possible to reach enough signal to noise to detect magnitude $30 \mathrm{AB}$ sources in the Ly $\alpha$ emission line. The satellite is being completed as of this writing. Launching is planned for the end of 2018.

The near IR camera would have a telescope with a $19 \mathrm{~cm}$ diameter primary mirror. This will allow a 50 degree survey field. This large field avoids problems with cosmic variance. Assuming a quantum efficiency of 35

\section{Summary}

We are planning to send a micro-satellite, carrying a near Infrared camera, to observe the first sources to appear in the Universe at a redshift of about 10. Our theoretical semi-analytical models predict that a first re-ionisation will have been occurred at about $\mathrm{z}=10$, achieved by PopIII stars. Hence there is a window to see the first galaxies that formed around that time. These galaxies would be ones that are responsible for completing the re-ionisation of the Universe by $\mathrm{z} \sim 5.5$.

\section{References}

[1] Bouwens, R. J., Illingworth, G. D., Oesch, P. A., et al. 2010, Ap. J. 709, L133 
[2] Bouwens, R. J., Illingworth, G. D., Oesch, P. A., et al. 2015, Ap. J. 803, 34

[3] Dressler A., Martin C. L., Henry A., Sawicki M., McCarthy P.,2011, ApJ, 740, 71

[4] Dressler A., Henry A., Martin C. L., Sawicki M., McCarthy P.,Villanueva E., 2015, ApJ, 806, 19

[5] Ellis R. S., McLure R. J., Dunlop J. S., et al., 2013, ApJ, 763, L7

[6] Erb D. K., 2015, Nature, 523, 169

[7] Fan, X. , Strauss, M. A., Becker, R. H., et al. 2006, Ap. J. 132, 117

[8] Giavalisco M., Ferguson H. C., Koekemoer A. M., et al., 2004, ApJ, 600, L93

[9] Haiman Z., Spaans M., 1999, ApJ, 518, 138

[10] Henry A. L., Martin C. L., Dressler A., Sawicki M., McCarthy P., 2012, ApJ, 744, 149

[11] Meier D. L., 1976, ApJ, 207, 343

[12] McLure R. J., Dunlop J. S., Cirasuolo M., et al., 2010, MNRAS, 403, 960

[13] Ouchi M., Shimasaku K., Furusawa H., et al., 2003, ApJ, 582, 60

[14] Ouchi M., Shimasaku K., Akiyama M., et al., 2008, ApJS, 176, 301

[15] Ouchi M., Shimasaku K., Furusawa H., et al., 2010, ApJ, 723, 869

[16] Partridge R. B., Peebles P. J. E., 1967, ApJ, 147, 868

[17] Shapley A. E., Steidel C. C., Pettini M., Adelberger K. L., 2003, ApJ, 588, 65

[18] Steidel C. C., Adelberger K. L., Giavalisco M., Dickinson M., Pettini M., 1999, ApJ, 519, 1

[19] Salvador-Solé, E. Manrique, A. GuzmÃąn, R. Rodrı'guez Espinosa, J.M., et al. 2017, Ap. J. 834, 49

[20] Taniguchi Y., Ajiki M., Nagao T., et al., 2005, PASJ, 57, 165

[21] Yan H.-J., Windhorst R. A., Hathi N. P., et al., 2010, Research in Astronomy and Astrophysics, 10, 867 